\title{
Beobachtungen über das foveale Sehen der total Farbenblinden.
}

\author{
Von \\ Prof. C. Mess (Würzburg).
}

I.

Bekanntlich wurde in den letzten Jahren zur Erklärung des Sehens der total Farbenblinden wiederholt die Annahme gemacht, dass hier lediglich Stäbchen in der Netzhaut functionsfähig seien, und man hat demgemäss die total Farbenblinden als "Stäbchenseher" bezeichnet.

Im Speciellen sind folgende vier Hypothesen aufgestellt worden :

1. Es soll Jediglich Mangel oder Functionsunfähigkeit des Zapfenapparats vorliegen, während die sonstigen Verbältnisse, insbesondere die räumliche Vertheilung der Stäbchen, mit der Norm übereinstimmen.

2. Es sollen überall statt der Zapfen Stäbchen gebildet sein.

3. Es sollen den supponirten fovealen, an Stelle der Zapfen gebildeten Stäbchen andere Leitungsverbältnisse zukommen als den extrafovealen.

4. Es sollen diese fovealen Stäbchen einen geringeren Sehpurpurgehalt besitzen als die extrafovealen.

Die erste Hypothese ist widerlegt durch den Nachweis, dass bei mehr als der Hälfte der bisher untersuchten total Farbenblinden trotz eifrigsten Suchens eine der Fovea entsprechende blinde Stelle nicht nachgewiesen bezw. das Vorhandensein einer solchen sicher ausgeschlossen werden konnte ${ }^{1}$ ).

Es besteht auf manchen Seiten noch eine gewisse Neigung, für jene Fälle, wo ein centrales Skotom nicht nachgewiesen werden

1) Vgl. Hess und Hering, Untersuchungen an total Farbenblinden. Pflüger's Arch. Bd. 71 S. 105. - Hess, Weitere Untersuchungen über totale Farbenblindheit. Zeitschr. f. Psych. u. Physiol. Bd. 29 S. 99. - Hess, Ueber den Ablauf des Erregungsvorgangs u. s. w. Arch. f. Ophthal. Bd. 51 (2) S. 225. 
konnte, die Möglichkeit einer nicht genügend genauen Untersuchung zu betonen. Demgegenüber möchte ich folgende vier Punkte hervorheben:

Erstens konnte in den sechs von Hering und von mir untersuchten Fällen eine deutliche foveale Minderempfindlichkeit bei berabgesetzter Beleuchtung nachgewiesen werden, die bei hellerer Beleuchtung nicht vorhanden war. Diese Beobachtung lässt sich schwer mit der Annahme vereinigen, dass hier ein centraler Gesichtsfeldausfall vorhanden, aber nicht nachweisbar gewesen sei.

$\mathrm{Z}$ weitens scheinen mir manche der üblichen Methoden, deren man sich zum Nachweise eines fovealen Gesichtsfeldausfalles zu bedienen pflegt, durchaus nicht so einwandfrei, als vielfach angenommen wird. Wenn man gar das schlechte Sehen der total Farbenblinden im hellen Lichte auf hochgradige locale Adaptation infolge leichter Ermüdbarkeit der Stäbchen bezieht ${ }^{1}$ ) (wie thatsächlich geschehen ist), wird man besondere Bedenken tragen müssen, aus dem Unsichtbarwerden kleiner Sehobjecte bei so herabgesetzter Sehschärfe ohne Weiteres auf einen entsprechenden Gesichtsfeldausfall zu schliessen. Dass für die vorliegende Frage solchen Defecten keine grosse Bedeutung zugeschrieben werden kann, die sich nur nachweisen lassen mit Sehobjecten, deren Flächeninhalt we niger a ls $1 / 140$ von dem dem normalen stäbchenfreien Bezirke entsprechenden beträgt, bedarf keiner Erörterung.

Wie vorsichtig man in der Beurtheilung des Nachweises eines centralen Skotoms überhaupt sein muss, zeigt u. A. die Erfahrung, dass noch vor wenigen Jahren die Behauptung aufgestellt werden konnte, die Fovea des normalen Auges sei blaublind, habe also ein Skotom für Blau (das, auf $1 \mathrm{~m}$ Abstand projicirt, einen Durchmesser von $17-20 \mathrm{~mm}$ haben sollte), und dass für den Nachweis eines solchen angeblichen Skotoms die Beobachtungen an den Augen mehrerer anerkannt ausgezeichneter und sorgfältiger Beobachter angeführt werden konnten.

Im Hinblicke auf eine unrichtige Wiedergabe meiner Arbeiten sei ausdrücklich betont, dass ich die Möglichkeit des Vorkommens eines centralen Gesichtsfeldausfalles beim total Farbenblinden niemals

1) Die gegen eine- solche Annahme zu erhebenden Bedenken habe ich früher eingehender erörtert. 
bestritten und mehrfach ausdrücklich betont habe, es könne einem solchen eine Bedeutung für das Verständniss der totalen Farbenblindheit nicht zukommen.

Drittens: Da man bei Erörterung der Schwierigkeiten der Untersuchung auf foveales Skotom gerne den "unüberwindlichen" Nystagmus betont, sei darauf hingewiesen, dass ich schon vor mehreren Jahren eine einfache (auf Momentbelichtung beruhende) Methode angegeben habe ${ }^{1}$ ), mit deren Hülfe der störende Einfluss des Nystagmus sich sehr leicht überwinden lässt. Ich habe mit dieser Methode auch neuerdings wieder bei der schon früher von mir untersuchten Frl. M. Untersuchungen angestellt, welche einen weiteren Beweis für das Fehlen eines centralen Skotoms bei ihr erbrachten.

Viertens sei hier eine neue Beobachtung angeführt, die in besonders eindringlicher Weise das foveale Sehen total Farbenblinder beweist.

Die zuerst vor drei Jahren von mir untersuchte ${ }^{2}$ ), jetzt 23jährige, total farbenblinde Frl. M. kann mit ihrem linken Auge (leichte Myopie mit Astigmatismus, $S=1 / 5$ ) genügend fixiren, um auch von kleineren hellen Objecten kräftige Nachbilder zu erhalten. In der Mitte eines grossen mattschwarzen Cartons waren sieben kreisrunde Löcher von je $6 \mathrm{~mm}$ Durchmesser so ausgeschlagen, dass sechs in Form eines regelmässigen Sechseckes um den mittleren, siebenten angeordnet waren. Der Carton war über eine vertical stehende, von rückwärts passend beleuchtete Milchglasplatte gespannt. Die äusseren Ränder je zweier einander gegenüber liegender Löcher standen um $36 \mathrm{~mm}$ von einander ab. Fixirte die Patientin aus einem Abstande von $120 \mathrm{~cm}$ von der schwarzen Fläche das in Augenhöhe befindliche mittlere Scheibchen, so bildeten sich, wenn man die üblichen Maassangaben für den Durchmesser des stäbchenfreien Bezirks zu Grunde legt, sämmtliche sieben Scheibchen vollständig auf stäbchenfreiem Gebiete ab. Die Dame fixirte gleichzeitig mit einer normalen Vergleichsperson während 10 Secunden die Mitte der Figur; nach Verdecken der Augen sah sie ungefähr gleichzeitig mit dem Normalen sieben von hellen Lichthöfen umgebene dunkle, runde Flecke, die für sie ungefähr ebenso lange

1) Vgl. Zeitschr. f. Psychol. u. Physiol. der Sinnesorgane Bd. 29 S. 100.

2) Arch. f. Ophthal. Bd. 51 (2) S. 248. 
sichtbar blieben wie für uns. Uebereinstimmend mit uns gab sie auch an, dass das mittlere und die excentrischen Scheibchen zuweilen nicht gleichzeitig auftauchten bezw. verschwanden. Der Versuch wurde öfter mit gleichem Ergebnisse wiederholt. Er zeigt, dass total Farbenblinde von Sehobjecten, die vollständig auf fovealem Gebiete abgebildet werden, ähnliche oder gleiche und gleich lang dauernde Nachbilder erhalten können wie der Normale.

Die oben unter Nr. 2 angeführte Hypothese, wonach beim total Farbenblinden überall statt der Zapfen Stäbchen gebildet sein sollen, muss in dieser allgemeinen Fassung zunächst die Vermuthung erwecken, dass die supponirten fovealen Stäbchen wenigstens das einzige bisher sicher festgestellte physiologische Merkmal der Stäbchen zeigen würden, nämlich die Fähigkeit, im Finstern Sehpurpur anzuhäufen, auf welche ja die ihnen zugeschriebene besonders hohe Adaptationsfähigkeit bezogen wird.

Eine derartige Hypothese ist ausgeschlossen durch den Nachweis, dass bei den von uns untersuchten total Farbenblinden, ganz so wie beim Normalen, entsprechend der Stelle des directen Sehens sich ein Netzhautbezirk fand, der im dunkeladaptirten Auge für schwache Lichtres ${ }^{-a}$ relativ weniger erregbar war als die umgebenden Netzhautpartien.

Bei der eben erwähnten Fräulein M. habe ich neuerdings abermals eine grössere Reihe von Beobachtungen angestellt, welche diese foveale Minderempfindlichkeit des dunkeladaptirten Auges schlagend darzuthun gestatteten.

Die oben unter Nr. 3 angeführte Hypothese ist schon widerlegt durch den Nachweis, dass die fragliche foveale Minderempfindlichkeit sich nur im dunkeladaptirten Auge findet, dagegen im helladaptirten fehlt.

Die vierte oben angeführte Hypothese stellt eine besondere Kategorie von percipirenden Elementen auf, durch welche der bisher so scharf betonte Unterschied zwischen Stäbchen und Zapfen hinsichtlich der Purpurbildung ganz verwischt wird. Das einzige bis jetzt bekannte physiologische Merkmal der Stäbchen ist ihre Fähigkeit, nachweisbar Sehpurpur zu bilden, während den Zapfen diese Fähigkeit nicht, wenigstens nicht nachweisbar, zukommt. Nun wird hier eine besondere Art von Elementen angenommen, die man Stäbchen nennt, obschon ihnen jenes einzige physiologische Merk- 
mal der Stäbchen mehr oder weniger vollständig fehlen soll; denn sie würden sich hinsichtlich der Purpurbildung ungefähr so, wie die fovealen Zapfen des normalen Auges verbalten. Man fragt vergebens nach einem Grunde für die Aufstellung dieser Kategorie von Elementen. Der Umstand, dass die fraglichen Gebilde nur die Empfindung farbloser Helligkeit vermitteln, kann unmöglich als Grund von Seiten jener Autoren angeführt werden, die schon für das normale Auge (auf der peripheren Netzhaut) das Vorkommen nur farblose Empfindungen vermittelnder Zapfen annehmen. Es ist eine seltsame Hypothese, die annimmt, dass total farbenblinde Zapfen nurim normalen Auge vorkommen, nicht aber im total farbenblinden.

Das Gesagte dürfte zur Charakterisirung der oben unter Nr. 4 angeführten Hypothese genügen. -

Die Anschauungen, zu welchen ich selbst durch die Untersuchung einer Reihe total Farbenblinder geführt wurde, sind bekanntlich mehrfach stark entstellt und verstümmelt wiedergegeben worden; wiederholt hat man mir gerade das Gegentheil von dem, was ich wirklich gesagt habe, in den Mund gelegt. Es erscheint daher nothwendig, meinen Standpunkt nochmals in Kürże klarzulegen.

Die alte, von Max Schultze begründete und wesentlich von Kühne, Haab und Parinaud ausgebildete Lehre, wonach die Stäbchen lediglich farblose, die Zapfen daneben auch farbige Empfindungen vermitteln, wird durch unsere Untersuchungen in keiner Weise berührt. Es steht in directem Widerspruche mit den Thatsachen, wenn behauptet wird, die Hering'sche Schule verhalte sich gegen diese Anschauungen ablehnend. So wurde z. B. sehon in der ersten von Hering gemeinsam mit mir veröffentlichten Abhandlung aber das Sehen der total Farbenblinden ausdrücklich' betont, dass diese nun beinahe 40 Jahre alte Ansicht $^{1}$ ) wohl mit der 'Theorie

1) Wie verbreitet die Schultze'schen Anschauungen auch in den $70 \mathrm{er} u$. $80 \mathrm{er}$ Jahren waren, möge nur an folgenden zwei Citaten gezeigt werden, die ich Herrn Geh.-Rath Hering verdanke. In der 3. Aufl. der Allgem. und mikroskopischen Anatomie von W. Krause (1876) werden die percipirenden Elemente der Netzhaut als "Stäbchen- oder Lichtzellen" und als "Zapfen-oder Farbenzellen" unterschieden. In einer Dissertation von Butz aus Rählmann's Klinik „Ueber die physiologischen Functionen der Peripherie der Netzbaut" (Dorpat 1888) wird es als eine "allgemein verbreitete" Ansicht bezeichnet, dass den Zapfen die Farbenempfindung zu Grunde liege. Es steht 
der Gegenfarben in Einklang steht, nicht aber mit der Dreifasertheorie; und in einer anderen Abhandlung habe ich diese Ansichten ausdrücklich wohl discutirbar genannt. Alle meine Ausführungen richteten sich, wie ich stets ausdrücklich betont habe, nur gegen die andere, von jener allgemein bekannten Schultze'schen ganz unabhängige Hypothese, nach welcher der Erregungsvorgang in den Zapfen sich gemäss der Dreifasertheorie abspielen soll, und gegen die Consequenzen, die man für die vorliegenden Fragen aus jener Hypothese gezogen hat.

II.

Die Unzulänglichkeit der eingangs angeführten Hypothesen über das Sehen der total Farbenblinden ist durch das Vorstehende schon genügend dargethan. Da man sie aber auch neuerdings zu vertheidigen versucht hat, so schien es mir erwünseht, die Frage nach dem Sehen - insbesondere dem fovealen - solcher Farbenblinder noch von einer neuen Seite in Angriff zu nehmen.

Bisher war das einzige für unsere Untersuchungen zu verwerthende Merkmal, durch das sich die fovealen Elemente von den extrafovealen unterscheiden, die geringere Adaptationsfähigkeit der ersteren, und es zeigte sich, dass hierin der total Farbenblinde sich nicht anders verhält, als der Normale. Neuerdings haben wir aber zwei weitere charakteristische Eigensehaften der fovealen Elemente im normalen Auge kennen gelernt, die zur Untersuchung an total Farbenblinden gut geeignet schienen. Es handelt sich um die von mir beobachtete Thatsache, dass die nach kurzdauernder Reizung des normalen Selorgans mit mässig hellem Lichte auftretende zweite helle Phase des Abklingens der Erregung (= Phase 3$)^{1}$ ) foveal wesentlich später auftritt als extrafoveal, und zwar um so später, je näher die gereizte Stelle der Mitte des stäbchenfreien Gebietes liegt. Sehr schön ist die Erscheinung schon $\mathrm{zu}$ sehen, wenn man einen etwa $6-8 \mathrm{~mm}$ breiten, passend belichteten mattweissen Cartonstreif über einem dunklen Hintergrunde mässig schnell vor dem ruhig gehaltenen Auge

in Widerspruch mit den Thatsachen, wenn behauptet wird, die Lehre M. Schultze's habe gerade bei denjenigen Physiologen, die sich speciell mit der Theorie des Farbensehens beschäftigten, wenig Beachtung gefunden.

1) Vgl. Hess, Untersuchungen über das Abklingen der Erregung nach kurzdauernder Reizung des Sehorgans. Pflüger's Arch. Bd. 95 S. 1. 
vorüberführt. Die fragliche Phase 3 ist dann als ein zum Reizstreif paralleler Nachbildstreif sichtbar, der entsprechend der Stelle des directen Sehens deutlich nach rückwärts ausgebuchtet und etwas schmäler erscheint, als extrafoveal; die Form der Ausbuchtung gibt gewissermaassen graphisch die Reactionsgeschwindigkeiten der einzelnen Punkte des fovealen Gebietes wieder.

Weiter konnte ich nachweisen, dass in dem gut dunkeladaptirten Auge bei passender Herabsetzung der Lichtstärke des (farblosen) Reizlichtes auch die Pbase 1 der Erregung bei kurzdauernder Reizung foveal wesentlich später auftitt und kürzer dauert, als extrafoveal ${ }^{\mathbf{1}}$ ). Bewegt man den geraden, weissen, sehr schwach belichteten Streif auf dunklem Grunde an dem dunkeladaptirten Auge vorüber, so erscheint dieser Streif nicht gerade, sondern foveal deutlich - ganz ähnlich, wie ich es für Phase 3 beschrieben habe - nach hinten ausgebuchtet, schmäler und weniger hell als extrafoveal. (Von der dritten Phase ist bei dieser geringen Lichtstärke des Reizlichtes nichts zu sehen.)

Auch der Ungeübte nimmt in der Regel die hier geschilderten Erscheinungen bald in ihrer charakteristischen Form wahr, und es konnte daher mit Aussicht auf Frfolg an die Untersuchung total Farbenblinder nach diesen Methoden geschritten werden, die für die in Rede stehenden Fragen wichtige Ergebnisse versprach. Bisher konnte ich an drei total Farbenblinden ein auch in dieser Hinsicht mit dem Normalen übereinstimmendes Verhalten nachweisen.

Die 52 jährige Frau L. $(S=6 / 60-6 / 36$ mit $+6 D$; kein merkbarer Nystagmus) sah sofort bei den ersten Versuchen den der Phase 3 entsprechenden bellen Nachbildstreif und gab nach einigen Beobachtungen mit voller Bestimmtheit an, dass derselbe in der Mitte eine Ausbuchtung nach rückwärts zeige, die nach ihrer Schilderung offenbar sich jener in unseren eigenen Augen ähnlich oder gleich verhielt. Die Versuche wurden häufig mit gleichem Erfolge angestellt und von anwesenden Collegen controlirt. (Auf die Ausbuchtung der Pbase 1 wurde, da diese mir damals noch nicht bekannt war, hier nicht untersucht.)

Eine zweite Beobachtungsreihe nahm ich an der schon erwähnten 23jäbrigen Fräulein $M$ vor. Nachdem sie sehr bald bei Be-

1) Genaueres hierüber wie über einige andere hierher gehörige Beobachtungen werde ich demnächst an anderer Stelle mittheilen. 
wegung des Papierstreifs den hellen Nachbildstreif wahrgenommen hatte, stellte ich ihr ohne weitere Erläuterung die Aufgabe, zu ermitteln, ob der letztere sich ihr als einfache gerade Linie darstelle oder nicht. Die Dame gab bald an, dass der Nachbildstreif in der Mitte eine deutliche Ausbuchtung nach rückwärts zeige, die bei langsainer Bewegung des Reizstreifs flacher erscheine als bei rascher. Bei zahlreichen Versuchsreihen mit mannigfacher Aenderung der Breite und Lichtstärke der Reizstreifen wie auch der Lichtstärke des Grundes wurden stets Ergebnisse erhalten, welche mit jenen an urseren normalen Augen (bis auf die Färbung) vollkommen übereinstimmten.

Ein Versuch, die Grösse der ausgebuchteten Partie zu schätzen, wurde in der Weise vorgenommen, dass auf dem $4 \mathrm{~m}$ entfernten dunklen Grunde zwei weisse Marken in wechselndem gegenseitigem Abstande angebracht und so lange gegen einander verschoben wurden, bis die auf die Mitte zwischen beiden Marken blickende Dame angab, dass die Anfänge der Ausbuchtung ungefähr in der Gegend jener weissen Punkte zu liegen schienen; auch hier ergaben sich gleiche Werthe, wie für das normale Auge; der fragliche Abstand wurde, ebenso wie für mich ${ }^{x}$, etwas grösser gefunden, als dem Durchmesser des stäbchenfreien Bezirks nach den üblichen Maassangaben entspricht. Als ich der Dame dann eine Abbildung des fraglichen Nachbildverlaufes für den Normalen (Fig. 2 meiner vorher angeführten Arbeit) vorlegte, erklärte sie, dass sie die Erscheinung ganz so sehe, wie sie dort wiedergegeben sei.

Mit einem kurzen rechteckigen Papierstreif sah die Dame leicht aucb die von mir früher beschriebene langdauernde helle Phase (= Phase 5), die bei öfter wiederholten Versuchen für sie ganz oder nahezu ebenso lange bestehen blieb, wie für mehrere normale Vergleichsaugen. Ferner sah sie bei passend gewähltem Grunde leicht, dass die Phase 4 deutlich dunkler war als der Grund, über dem die Bewegung erfolgte.

Fast noch leichter und einfacher als der Nachweis der fovealen Verzögerung der Phase 3 gelingt meist jener der fovealen Ausbuchtung der Phase 1 für das dunkeladaptirte Auge. Sowohl Fräulein M. als auch die früher wiederholt von uns untersuchte, jetzt 36 jährige Fräulein F. (die ich kürzlich gemeinsam mit Herrn Collegen Tschermak daraufhin untersuchen konnte) gab schon

1) Vgl. l. c. S. 468. 
nach wenigen Versuchen an, dass der im Dunkelzimmer am Auge vorübergeführte, schwach belichtete weisse Streif nicht geradlinig erscheine, vielmehr in der Mitte deutlich - offenbar ähnlich oder ganz so wie für uns - nach rückwärts ausgebuchtet.

Ferner konnte ich noch eine bisher nicht untersuchte Patientin wenigstens auf das fragliche Verhalten von Phase 1 und 3 prüfen. Die. 37 jährige Dame, seit Geburt total farbenblind (die v. Hippelschen Gleichungen gelten angenähert auch für ihre Augen) trägt seit ihrem fünften Jahre Convexgläser von 7 Dioptr. und kann damit leidlich in die Ferne sehen und bequem lesen; eine genauere Refractions- und Astigmatismusbestimmung war, da ich die Dame auswärts, ohne genügende Hülfsmittel, untersuchen musste, nicht möglich; nach einer Bestimmung mit Landolt's Ringproben beträgt die Sehschärfe mit ihren Gläsern $\overline{5}^{6 / 60}$. Es besteht mässige Lichtscheu und Nystagmus. Sehnerv normal; auch am übrigen Augenbintergrunde lassen sich krankhafte Veränderungen nicht nachweisen (doch musste ich mich auf eine nicht sehr eingehende Untersuchung ohne künstliche Pupillenerweiterung beschränken). Bei Untersuchung: auf Skotom mit der von mir angegebenen Methode (siehe oben) macht die Dame stets richtige Angaben; ein centraler Defect von irgend nennenswerther Ausdehnung ist also ausgeschlossen.

Bei Untersuchung mit bewegtem weissem Streif nimmt sie sowohl bei der Phase 1 wie bei der Phase 3 die foveale Ausbuchtung bald mit Sicherheit wahr.

Nach Abschluss dieser Untersuchungen erhielt ich durch die Güte des Herrn Collegen Tschermak noch über einen vierten Fall Bericht. Es handelt sich um einen (bis dahin noch nicht genauer untersuchten) 20jährigen Studenten, der die bekannten charakteristischen Merkmale der übrigen von Geburt total Farbenblinden zeigt (Genaueres hierüber wird event. an anderer Stelle mitgetheilt). Bei Untersuchung mit bewegtem Streif sieht er die foveale Ausbuchtung der Phase 1 bei dunkeladaptirtem Auge, sowie jene der Phase 3 bei relativ helladaptirtem "ausserordentlich deutlich". Der Mittheilung war eine von dem Patienten selbst angefertigte Skizze beigefügt, aus der hervorgeht, dass er die Erscheinung offenbar ganz ähnlich oder genau so wie wir sieht. -

1) i. c. S. $8 \mathrm{f}$. und Tafel I Fig. 1. 
Man hat bekanntlich die Behauptung aufgestellt, bei dem total Farbenblinden bestehe eine sehr lange Nachdauer der Reize. Ich habe dies schon früher als unrichtig nachgewiesen; auch die hier mitgetheilten neuen Beobachtungen bei Fräulein M. zeigen, dass für sie weder nach kürzer noch nach länger dauernder Reizung, weder bei geringen noch bei hohen Lichtstärken die Nachbilder merklich länger dauern als für den Normalen.

Wir begegnen ferner immer wieder der von mir schon wiederholt als irrig nachgewiesenen Behauptung, dass beim Normalen wie beim total Farbenblinden auf eine kurzdauernde Reizung eine "Doppelerregung im Stäbchenapparate" erfolge. Diese Behauptung ist in dreifacher Beziehung unrichtig: Erstens hat man bei den fraglichen Beobachtungen stets den der Zeit nach weitaus grössten Theil des ganzen Nachbildverlaufes vollständig übersehen (nämlich die von mir als Phase 4,5 und 6 beschriebenen Phasen); man müsste demgemäss mindestens von drei zeitlich auseinanderfallenden Empfindungseffecten, einer dreifachen Erregung, sprechen, denn es sind nach kurzdauernder Reizung mit mässig hellem Lichte drei helle Phasen des Nachbildverlaufes sichtbar. Zweitens handelt es sich bei dem fraglichen Vorgange nicht um zwei Erregungen, sondern um das phasische Abklingen einer einzigen Erregung, um positive und negative Phasen, d. h. solche, die zum einen Theile (Phase 1, 3,5) beller, zum anderen Theile $(2,4,6)$ dunkler sind als der (passend gewählte) Grund; es ist selbstverständlich nicht angängig, lediglich die hellen Phasen als Empfindungseffect oder Ausdruck der Frregung anzusprechen. Drittens ist es nicht zulässig, den der Phase 3 entsprechenden farblosen Theil des fraglichen Vorganges als Stäbchenerregung aufzufassen; denn ich habe gezeigt, dass er in wesentlich ähnlicher Weise (nur etwas anderem Tempo) a ch auf dem stäbchenfreien fovealen Gebiete sich abspielt.

Die hier kurz mitgetheilten neuen Beobachtungen haben die Thatsache ergeben, dass der foveale Bezirk des total Farbenblinden sich nicht nur hinsichtlich der geringeren Lichtempfindlichkeit des dunkeladaptirten Auges, sondern auch hinsichtlich des charakteristischen verspäteten Auftretens der ersten und der 
474 C. Hess: Beob. üb. das foveale Sehen der total Farbenblinden.

dritten Phase des Erregungsablaufes nach kurzdauernder Reizung ganz soverhält, wie jenerim normalen Auge. Auch hier nehmen die Reactionsgeschwindigkeiten der einzelnen Punktedes fovealen Gebietes von der Mitte nach der Peripherie hin ganz allmählich zu, offenbar in ähnlicher oder gleicher Weise, wie beim Normalen. 